\title{
Cloud radio access network fronthaul solution using optimized dynamic bandwidth allocation algorithm
}

\author{
Ebude Carine Awasume ${ }^{1}$, Stephen Musyoski², ${ }^{2}$ italice Kalecha Oduol ${ }^{3}$ \\ ${ }^{1}$ Department of Electrical Engineering, Pan African University Institute for Science, Technology, and Innovation \\ (PAUISTI), Jomo Kenyatta University of Agriculture and Technology (JKUAT), Kenya \\ ${ }^{2}$ Department of Telecommunications and Information Engineering, Technical University of Kenya, Kenya \\ ${ }^{3}$ Department of Electrical and Information Engineering, University of Nairobi, Kenya
}

\begin{tabular}{|c|c|}
\hline Article Info & ABSTRACT \\
\hline Article history: & In order to address the challenges that have come with the exploding demand \\
\hline Received Mar 16, 2020 & for higher speed, traffic growth and mobile wireless devices, Mobile network \\
\hline Revised Jun 10, 2020 & radio access network. The merits of cloud based RAN includes the ease of \\
\hline Accepted Jun 25, 2020 & $\begin{array}{l}\text { infrastructure deployment and network management as well as the fact that } \\
\text { its performance are optimized and it is cost effective the merits of cloud }\end{array}$ \\
\hline Keywords: & $\begin{array}{l}\text { based RAN includes the ease of infrastructure deployment and network } \\
\text { management as well as the fact that its performance are optimized and it is }\end{array}$ \\
\hline Cloud radio access network & cost effective. Notwithstanding, cloud radio access network comes with so \\
\hline Dynamic bandwidth allocation & many strict requirements to be fulfilled for its fronthaul network. In this \\
\hline Passive optical network & Particular interest on the time division multiplex passive optical network's \\
\hline Time division multiplex & $\begin{array}{l}\text { challenge of latency was treated by proposing an optimized version of the } \\
\text { round robin dynamic bandwidth allocation algorithm. Results obtained show } \\
\text { an improvement in the latency of the original algorithm which meets the } \\
\text { fronthaul requirement. Other test parameters like jitter and BER were also } \\
\text { improved by our proposed optimized algorithm. }\end{array}$ \\
\hline
\end{tabular}

This is an open access article under the $\underline{C C B Y-S A}$ license.

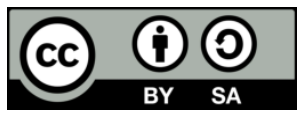

\section{Corresponding Author:}

Ebude Carine Awasume

Department of Electrical Engineering

Pan African University Institute for Science, Technology, and Innovation (PAUSTI)

Jomo Kenyatta University of Agriculture and Technology (JKUAT) Kenya

PAUSTI Complex block B, P.O BOX 62000-00200, Nairobi, Kenya

Email: ebude.carine@students.jkuat.ac.ke

\section{INTRODUCTION}

Telecommunications companies are constantly being challenged due to the drastic increase in data traffic as well as the desires of the users to experience higher data rate in using their products and getting services through diverse and ever dynamic network applications and request. Consequently, the mobile network has evolved to rise to the challenge by increase in coverage area of the network, network capacity as well as other quality of service (QoS) requirement. One of the most significant way of achieving this is adopting cloud radio access network (C-RAN) for small cell networks, a concept which has experience a significant interest from the industry recently [1]. There are early signs that this could be a promising RAN architecture for future generation of wireless network communication that could handle the increasing mobile traffic and meet the challenges mobile operators face with traditional RAN architecture. According to the operators, RAN is considered the most essential component to deliver the desired always available high QoS and speed for the users [2]. Mobile operators are always challenged to realize less expensive RAN 
technologies that can meet the increasing mobile traffic demand, increase QoS, and manage large number of complex devices [3].

In order to provide ubiquitous services and improve quality, emphasis is mostly laid on the RAN. The RAN architecture has evolved from the traditional RAN in which the base station is responsible for the functions of radio and baseband processing and the antenna sited closed to the radio [4] to the distributed RAN (D-RAN) which involves the separation of the base station into remote radio unit (RRU), digital unit (DU) and the signal processing unit (SPU) [5]. In the D-RAN, only one RRU is being served by a DU. The approach of C-RAN has shown through experiments, to save both operational expenditure (OPEX) and capital expenditure (CAPEX) [6].

There is separation of DU or BBU from the remote radio head (RRH) which represent the radio unit in the C-RAN architecture. This is as presented in Figure 1. Several RRHs share a BBU from the central cloud [7]. There are 3 essential components in the C-RAN architecture [8] as shown in Figure 1. These are:

- Several BBUs which forms a BBU pool with the centralized processors

- $\quad$ RRHs with antennas sited at remote sites

- The connection between RRHs to BBUs known as fronthaul which is characterized by low latency and high capacity.

The advantages of the architecture of C-RAN are ease of infrastructure deployment and network management as well as the fact that its performance are optimized and it is cost effective. The transport system will have stringent performance requirement in the order of tera bit per seconds (Tbps) to handle the huge number of radio channels, the joint processing of the several RRHs as well as fronthaul estimation [1]. There is now the need to address all the strict requirements of this fronthaul part of the network.

Fronthaul link is an essential feature of the C-RAN as it connects the RRUs to the centralized functions. C-RAN are tipped to be deployed in scenario where the users' density is high. Common public radio interface (CPRI) is an example of a common semi-proprietary interface which depends on time-domain samples of radio waveforms [9]. Data rates for CPRI differs greatly with the least being that of option 1 with rates of $614.4 \mathrm{Mbps}$ to the highest which is option 10 with data rate in region of $24.33024 \mathrm{Gbps}$. These rates are expected to be delivered between RRHs and BBUs covering several kilometers. It is for this reason that an appropriate fronthaul solution that will meet the requirements for a $5 \mathrm{G}$ network needs to be established. An optimized round robin dynamic bandwidth allocation algorithm is implemented in an NG-PON 2 fronthaul solution to be able to meet these requirements. Results measured using the parameters latency, jitter and BER showed an improvement.

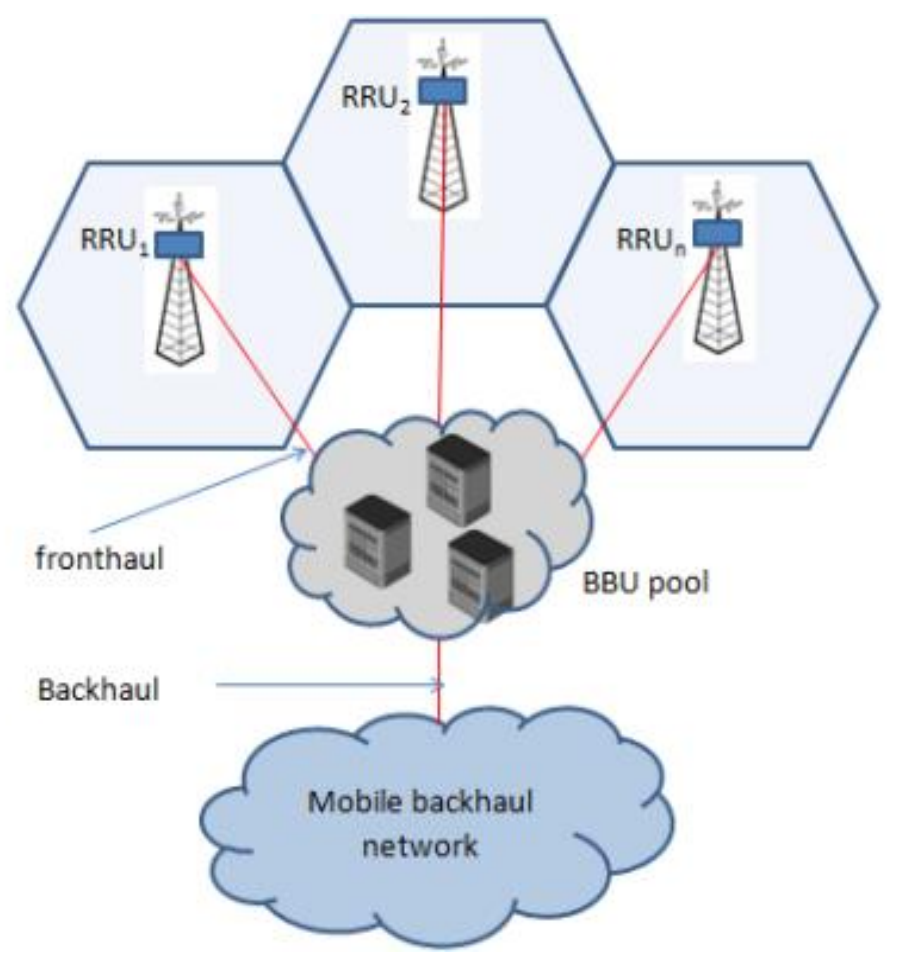

Figure 1. C-RAN architecture [8] 
This remainder of paper has been partition into 4 sections. Presentation of different C-RAN fronthaul requirements for $5 \mathrm{G}$ network has been done in Section 2. Section 3 presents possible optical fronthaul solutions with particular interest in the TDM-PON solution. The challenges faced by this TDMPON solution are presented in section 4 with possible solutions to these challenges. In section 5, the original round robin algorithm and the proposed optimized version are presented. Section 6 presents the simulation parameters used and the analysis of the results obtained. This paper is concluded in section 7.

\section{C-RAN FRONTHAUL REQUIREMENTS}

The definition of different terminologies pertaining to the C-RAN fronthaul is presented first,

- Fronthaul link: This is the connection between the remote radio unit and the DU pool. Open radio interface (ORI), common public radio interface (CPRI), and open base station architecture initiative (OBSAI) are common instance of fronthaul protocols.

- Fronthaul network: This is the part of the network which transports the fronthaul links and connects the RRUs and DU pool. Wavelength division multiplexing (WDM) network is an example of fronthaul network.

- Fronthaul channel: This is the channel used to convey information related to the fronthaul.

- Fronthaul solution: This is the platform to actualize the fronthaul network. The solution ensures that the fronthaul network meet the requirement [10].

There is a significant overhead burden on fronthaul link in the C-RAN architecture. Depending on the kind of data compression technique used and the different cell site technology, the bit rate of the fronthaul can be in the orders of gigabits per second. Also, fronthaul wireless traffic varies with time and needs different bandwidths at different times of the day. Consequently, It is first important that the design of the MFH should support flexible bandwidth allocation as well as high-bandwidth capacity [11]. Also, the fronthaul traffic should be sent in packets and transmitted across conventional packet-switched networks, like Ethernet.

Furthermore, there is need for the MFH to cope with the stringent limit of delay in real-time signal processing. This is necessary because the IQ data carried over CPRI link is in digital form. Also, any fronthaul solution must support at least options 2, 3, 4, 5, 6 and 7 of CPRI. It is optional for the solution to support CPRI options 1 and 8 [10]. Additionally, low latency must be achieved at all times in order to support important C-RAN applications like the virtual migration of BBU and coordinated multipoint (CoMP). The least acceptable performance for eMBB and URLLC fronthaul end-to-end delay are $4 \mathrm{~ms}$ and $500 \mu$ s respectively. This includes the delay from the equipment as well as the time spent for propagation.

Added to the above, either electrical or optical multiplexing of the CPRI links must be possible with the MFH solution. The multiplexed signal will be sent to the BBU pool. Additionally, the multiplexing of the FH links must be transmittable on the same channel. Information about the O\&M in the FH domain could also be transmitted concurrently using the same channel or a different channel can be used. When it comes to the topology for the fronthaul network, a ring topology is preferred so that it can save fiber as much as possible. Also, a tree and a point-to-point topology can also be used in order for network deployment to be flexible.

Due to the exceptional network performance required of $\mathrm{FH}$, many researchers have investigated how to address the challenge of achieving the design of FH network that fits well to deliver 5G C-RAN. Most of the work agreed that for FH traffic and requirements to be met, higher functional splits than CPRI which needs very low latency and demands high capacity will need to be researched on [12].

Lastly, for the purpose of reliability and quality of service, the fronthaul solution should have a backup so that it switches once it experiences a fiber failure. This failure should not cause service interruption at the level of the application layer. Also, there is a requirement of less than $50 \mathrm{~ms}$ switch/recovery time for the FH link [10].

\section{FRONTHAUL FIBER SOLUTIONS}

Traditionally, optical fibers based on single mode fiber (SMF) and multi-core fiber (MCF) are usually used in the C-RAN fronthaul links [13]. Optical solutions that can be employed for mobile fronthaul can vary from a dedicated fiber to Wavelength based systems or PON systems.

\subsection{Dedicated fiber solution}

In this solution, there is a direct dedicated fiber connection that connect the RRHs and the BBU pool. This is usually achieved using Dark fiber in form of large installed fibers which are unused by a particular operator. In this design, there is a dedicated fiber link for each RRH to BBU pool. The modulation 
and demodulation of CPRI signal to optical carrier is done by optical transponders which are located at both sides of the channel to perform $\mathrm{E} / \mathrm{O}$ or $\mathrm{O} / \mathrm{E}$ conversion. This solution is straightforward and the latency is low because it only possesses delay due to propagation of light. However, it has the issue of many fiber links deployments. The solution also suffers from lack of flexibility and reliability compared to other solutions [11]. The expansion is also challenging due to the cost needed to deploy new fiber links and there is limitation to this [14].

\subsection{Optical transport network (OTN)}

G. 709 is the framework proposed by ITU-T which represents optical transport network (OTN) responsible for the transfer of CPRI signals transparently [15]. OTN encapsulates CPRI traffic which is further multiplexed on the fronthaul. Also, OTN has the ability to reduce effect of bit errors sensitivity of the link by adopting forward error correction (FEC) [1, 14]. This result in extending the reach to metro area to form metro optical networks. However, the features of OTN which includes FEC has caused the demerit of increased latency in the transfer of CPRI traffic.

\subsection{Wavelength based system}

The motivation behind this solution is to achieve a cost efficient network with very capacity. In addition, in order to transmit CPRI signals, the WDM presents adequate characteristics which makes it a prospective solution. Another wavelength based solution which is very reliable, simple and has low delay is the CWDM. The technology behind CWDM multiplexing is cheap and all these make is good both for metro and access networks. The easily adaptable nature of CWDM during its deployment makes it appropriate for mobile fronthaul as the sites for the deployment of RRHs are usually unpredictable. Because of the unpredictable nature of the location of the different RRHs, the adaptability of CWDM to outdoor deployment as well as its cost effectiveness and high throughput makes it a suitable solution for mobile fronthaul. CWDM has been assigned a fixed wavelength by ITU-T consisting of 18 channels and $20 \mathrm{~nm}$ of spacing in between each channel. This fixed wavelength does not encourage the use of CWDM for commercial purposes as network expansion and migration becomes difficult. In addition to that, the 18 fixed channels offered by CWDM will not be enough in locations where there are many RRHs.

This leads us to the DWDM which can be used as a fronthaul solution for a case where many RRHs want to transmit at the same time to the BBU pool. DWDM as the name implies is a denser version of CWDM hence is more spectrum efficient. A typical example of a DWDM has 160 channels for a $25 \mathrm{GHz}$ grid with $0.2 \mathrm{~nm}$ spacing between the channels. Because of this closer channel spacing between wavelengths of DWDM, the frequencies can be said to be more stable compared to the CWDM schemes. One of the main goals of C-RAN is a cost effective network. This means that the cost of transmitters and high temperatures that comes with the deployment of DWDM needs to be studies for this goal to be met [1].

\subsection{PON based system}

The PON based system is a practical solution because it is cost effective and is readily available for the transportation of fronthaul date in a small cell scenario [16, 17]. Since it uses fiber optics as medium of transmission, it can carry the huge fronthaul data of CPRI in an ultra-dense small cell network. It's simple, passive nature and readily available optical distribution network makes it a more preferable solution for MNOs. However, due to the optical splitters used in PONs for the separation and collection of optical signals, it can lead to increase in latency and also higher power consumption hence a reduction in cell radius. WDMPON and the TDM-PON are the versions of PONs that are budget friendly and feasible when being used in an ultra-dense scenario.

XG-PON, GPON and 10G-EPON are examples of a time division multiplexed-PON. These TDM examples cannot exceed $10 \mathrm{~Gb} / \mathrm{s}$ bit rates and so they are appropriate and cheap for dense small cell scenarios with bitrates lower than $10 \mathrm{~Gb} / \mathrm{s}$ but they are not in a scenario where the small cells are scanty [1]. As concerns latency, the downstream latency for TDM-PONs is good for MFH as it uses broadcast. The problem comes with the upstream latency which is usually in the order of milliseconds. The main cause of this latency increase in the upstream is the dynamic bandwidth allocation algorithm it uses for transmission. Therefore, the DBA algorithm needs to be improved upon for the TDM-PON to be made an appropriate MFH solution. This will be discussed in details in section 4 .

The WDM-PON come with the option of providing different wavelengths in order for different point-to-point links to be established. It presents benefits like low latency, high transmission rate and its flexibility with different protocols and transmission rates. With wavelength capacity of up to $24.3302 \mathrm{~Gb} / \mathrm{s}$, it makes it an attractive solution for CPRI transmission. Because the WDM-PON can use a single wavelength for the simultaneous duplex transmission of traffic makes it more spectrum efficient when it comes to fiber usage [18]. Because of the shared optical infrastructure due to multiplexing of signals from different ONUs, 
there arises the need to purchase very expensive equipment like WDM multiplexer/de-multiplexer and transceivers. This cost increase limits the implantation of this fronthaul solution.

A TWDM-PON is a hybrid implementation of the 2 above mentioned PON systems. An example of this hybrid PON is the NG-PON2. For this hybrid scheme to be deployed, it makes use of both wavelength and time multiplexing. The TDM makes available several PtMP links using a DWDM and on another DWDM, there are several free point-to-point links made [19]. The two multiplexing schemes functions using a shared optical distribution network (ODN), Figure 2 illustrates this. The NG-PON2 architecture is basically a stack of four XG-PONs. This makes it to be able to provide up to $40 \mathrm{~Gb} / \mathrm{s}$ aggregate in the downstream and $10 \mathrm{~Gb} / \mathrm{s}$ in the upstream. Apart from the latency challenge that comes with TDM-PON systems, there is also the problem of realizing low cost tunable filter at the level of ONUs.

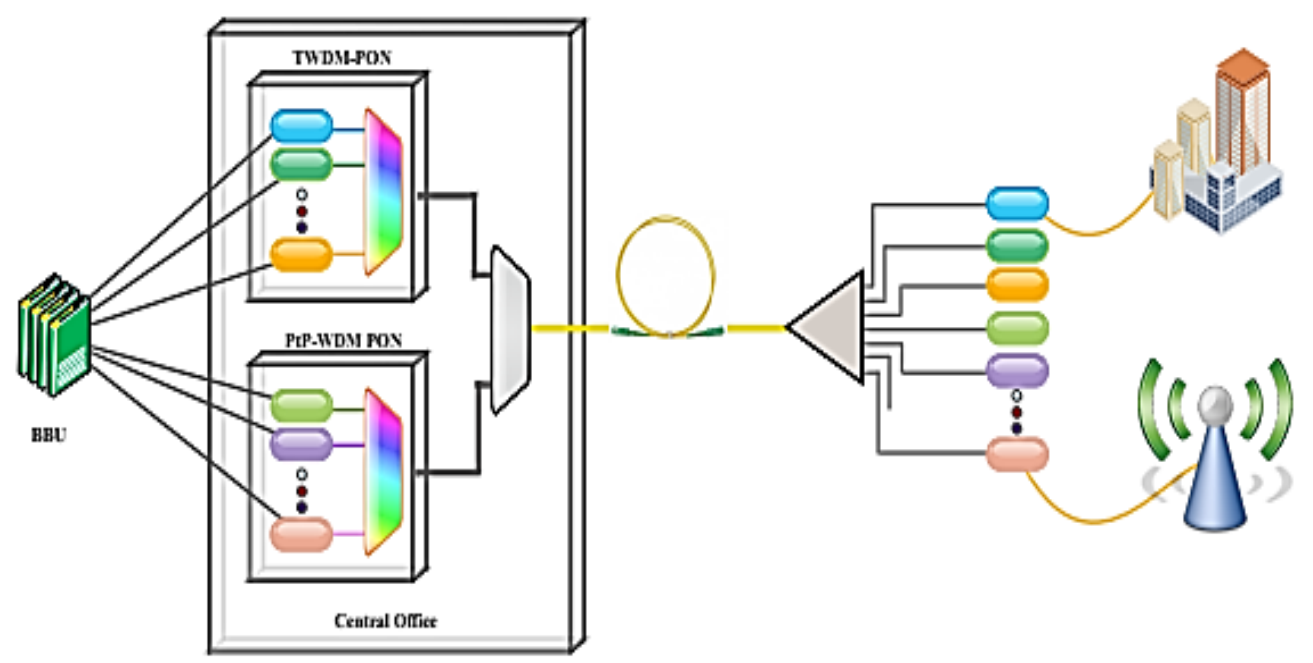

Figure 2. An overlay of DWDM-PON and TWDM-PON using same ODN [1]

\section{TDM-PON BASED FRONTHAUL CHALLENGES AND SOLUTIONS}

4.1. Challenges

TDM-PON as a fronthaul solution is a good option because of its simplicity and its commonly available optical distribution network. All these make it a very cost effective MFH solution. As a first concern with TDM-PON being a good fronthaul solution, we encounter the challenge of capacity and bandwidth [1, 15, 20]. Because the CPRI fronthaul interface used in C-RAN has very high bitrates, multiplexing these signals over an XG-PON channel of $10 \mathrm{~Gb} / \mathrm{s}$ is not a good solution. This challenge of capacity has been resolved because of ITU-T's G989 (NG-PON2) with a single channel of up to $40 \mathrm{~Gb} / \mathrm{s}$. Also, as a solution to capacity and bandwidth limitation, there has been a lot of research work on different compression techniques of CPRI signals before transmission. Progress has been made as there has been the compression between $2 \mathrm{X}$ and $3 \mathrm{X}$ of the original signal capacity.

In addition, for the uninterrupted transmission of CBR traffic in CPRI, there is the need for TDMPONs to have a quiet window so as to prevent transmission from new ONUs [15]. Also, there are additional requirements for the security and synchronization of CPRI. These additional requirements are not very essential in TDM-PONs because solutions already exist but are not treated in this paper. Apart from the above, latency is still a big problem with TDM-PONs. The latency requirement for $5 \mathrm{G}$ fronthaul should be $500 \mu \mathrm{s}$ but that of TDM-PONs is usually about $1 \mathrm{~ms}$ particularly in the upstream due to its DBA grant request sent to the OLT before transmission begins $[1,15,20-22]$.

\subsection{Solutions to TDM-PON fronthaul latency challenge}

\subsubsection{Fixed bandwidth allocation (FBA)}

FBA has been used as a solution to the high latency in TDM-PONs. By assigning fixed bandwidths between the OLT and its different ONUs. Because of the fixed bandwidths, control signals are not needed between the OLT and the ONU. Nevertheless, these fixed bandwidth assigned to every link is not bandwidth efficient with respect to statistical multiplexing making the bandwidth requirement to always remain high $[20,22-25]$ 


\subsubsection{Mathematical analysis using queuing theory}

Prasanth, Walid and Pfeiffer, used mathematical analysis to solve this latency problem. There was the use of the notion of queuing theory to mathematically analyse the overall delay. Then a study of the possible trade-off that could be made using several parameters were derived from closed-form formulas. At the end, in different application cases, a $250 \mu$ s of acceptable latency was established [15].

\subsubsection{Low latency DBA schemes}

Most of the efforts by researchers to reduce latency in TDM-PONs has been done by modification of the DBA algorithm. Mikaeil et al. [21] did so by optimizing the high latency round-robin DBA of XGPON. Zhou et al. [22] and Tashiro et al. [26] worked on a complex programming that uses the mapping of LTE RB to transmit its outcome to the TDM-PON. This helped to cut down on the extra delay in PON scheduling. Ou et al. [16] Modified latency reduction by applying transmission division to the DBA. Horvath et al. [27], reduced the latency of three essential services which are video, voice and data. In this work, different transmission containers (T-CNTs) were used to represent these three services. T-CONTs help improve a PON's DBA status.

In [21] performance comparison was done for two new XG-PON DBAs. These are the round-robin DBA (RR-DBA) and group-assured GIANT (gGIANT). They were compared using burst simulated mobile front-haul traffic. Neither of these DBAs was found to meet the delay requirements for a C-RAN fronthaul but the RR-DBA gave a better performance for upstream delay compared to the gGIANT. It is for this reason that we decide to optimize the RR-DBA instead of the gGIANT.

\section{RESEARCH METHOD}

Generally, the DBA procedure in PONs is by assigning upstream opportunities to different ONUs connected to the OLT [28]. This is because downstream transmission is done by broadcasting from the OLT to the different ONUs. Every ONU is being grouped in different QoS classes of service (CoS) and positioned in the appropriate T-CONT in order for the OLT to treat them separately [29]. These T-CONTS are either Fixed (T-CONT1), Assured (T-CONT2), Non-Assured (T-CONT3) or Best Efforts (T-CONT4).

Generally, the OLT continuously collects DBA reports and calculates the upstream traffic to assign to each ONU. The calculated results are sent to each ONU by a bandwidth map and the ONUs then transmit upstream data through the permitted timeslots in the map [30]. The difference between DBAs is in the manner in which the assigned upstream traffic is calculated for each ONU.

\subsection{Round-robin DBA algorithm}

The RR-DBA originates from an old, fair and simple algorithm that treats all T-CONTs equally by assigning them an amount of bytes less than or equal to the maximum allocation bytes $W_{\max }$ and in a circular manner. There is a processing time $T$ assigned to each ONU for each cycle given by

$$
T=\frac{C}{K}
$$

where $C$ represents the duration of a cycle and there are a total of $K$ ONUs in the network [27]. When the occupancy report $R_{t}^{j}$ from the $j t h$ T-CONT during the $t$ cycle reaches the OLT, the DBA mechanism allocates the grant $G_{t}^{j}$ as follows

$$
G_{t}^{j}=\left\{\begin{array}{rrr}
R_{t}^{j} \quad \text { if } & R_{t}^{j} \leq W_{\max } \\
& W_{\max } & \text { else }
\end{array}\right.
$$

where $W_{\text {max }}$ help to stop the over demanding T-CONTs from overusing the upstream bandwidth but this does not ensure bandwidth efficiency as some bandwidth will not be used.

\subsection{The optimized round-robin DBA algorithm}

Because of the bursty nature of fronthaul traffic, the use of maximum allocation bytes for every T-CONT as in RR-DBA will lead to latency increase in delay and upstream bandwidth inefficiency. This optimized RR-DBA algorithm will divide the excess bandwidth from the not heavy T-CONTs in a previous cycle to the highly demanding T-CONTs in the next cycle. At the beginning of the first cycle, all T-CONTs are assigned the maximum allocation bytes $W_{\max }$ and there are no excesses, i.e 


$$
W_{0}^{j}=W_{\text {max }}=\frac{\text { Total bandwidth }, B W}{\text { Number of } T-\operatorname{CONTS,K}}
$$

and $E x_{0}^{j}=0$

The grant allocation following the reception from the T-CONTs their different occupancy reports $R_{t}^{j}$ will as follows

$$
G_{o}^{j}=\min \left(R_{1}^{j}, W_{0}^{j}\right)
$$

By comparing the occupancy reports and the maximum allocation bytes, a Boolean variable $\mathrm{B}$ and counter $\mathrm{N}$ is updated i.e. if $R_{t}^{j}>W_{\max }, B==$ True, $N++$. At the end of the cycle, $\mathrm{N}$ will be the number of T-CONTs whose demand in teor occupancy report was higher than the maximum allocation bytes. The next step consists of calculating the new limit for upcoming cycle for heavily loaded T-CONTs (i.e. $B==$ True). The new limit will be $W_{1}^{j}=W_{\text {max }}+E x_{0}^{j}$ but if $B==$ False, the limit remains unchanged $W_{1}^{j}=W_{\text {max }}$. The excess bandwidths to be added to the heavily loaded T-CONTs will be calculated by

$$
E x_{t}^{j}=\frac{\left(B W-\sum_{J=0}^{K} W_{t}^{j}\right)}{N}
$$

This optimized algorithm assumes that the T-CONTs will maintain the same buffer occupancy for at least one upcoming cycle.

\section{RESULTS AND DISCUSSIONS}

The performance of our optimized algorithm was evaluated in comparison to the default RR DBA algorithm. For this, we used a discrete-event network simulator NS 3 and its module for NG-PON 2 to carry out several simulations. We created a cloud radio access network (C-RAN) consisting of 1 Cloud node, 200 $5 \mathrm{G}$ user nodes, $5 \mathrm{RRH}$ nodes (frequency of $20 \mathrm{MHz}, 3$ sectors and 4 antennas), 5 ONU (optical network units) and macro base station based on the ring topology. We constructed the network based on the NG-PON 2 for the efficient transmission. All users are connected to an RRH and RRHs are connected to ONUs. 5G small cell uplink traffic is generated using the poisson Pareto burst process (PPBP) and injected into each ONU.

Three key parameters were used to evaluate the performance of the optimized algorithm with respect to the original RR algorithm. These parameters are: Latency (which is the end-to-end delay used by packets in addition to the time spent in the equipment and time travelling in the fiber medium), Jitter (which is the rate of change of the latency) and BER.

Figure 3 in Appendix shows the different graphs where the optimized RR-DBA algorithm is compared to the default algorithm for the parameters of latency, jitter and BER with increasing ONU loads. The optimized algorithm outperforms the original algorithm for every parameter. For Figure 3(a), the latency of the optimized algorithm is better as it uses the excess bandwidth of the less heavy T-CONTs and hence reduces the waiting time. It can also be seen that the original algorithm fails to satisfy the requirement of $\leq$ $500 \mu$ s for a $5 \mathrm{G}$ fronthaul meanwhile the optimized algorithm satisfies this requirement between the 100 and $140 \mathrm{Mbps}$ interval which accommodates the throughput for a $20 \mathrm{MHz} /$ three sectors/four antennas when the MAC-PHY split is considered (123.2 Mbps).

For Figure 3(b), the optimized algorithm also outperforms the original algorithm as jitter is a variation of the latency: the change in the delay between packets which has been reduced. Also, as the load increases, the jitter too continues to increase. For Figure 3(c), the optimized algorithm presents a better BER than the original RR algorithm. This is due to the reduction in congestion of the T-CONTs in the optimized algorithm as it allows the bigger window of transmission is being used by the different T-CONTs.

\section{CONCLUSION}

In this study we presented an insight into the C-RAN fronthaul moving from the requirements to the different optical fronthaul solutions that can be used. Among the solutions presented, the TDM-PON was our point of interest and so challenges particular to it and proposed solutions were presented. We also proposed an optimized Round Robin algorithm which helps reduce the latency to satisfy the requirements of a 5G fronthaul network. An improvement in the jitter and BER parameters of the fronthaul network with the optimized algorithm was also noticed. Further work can also be done by evaluating different parameters like the packet delivery ratio and throughput using this optimized round robin algorithm. 


\section{APPENDIX}

$1 . \mathrm{E}+02$

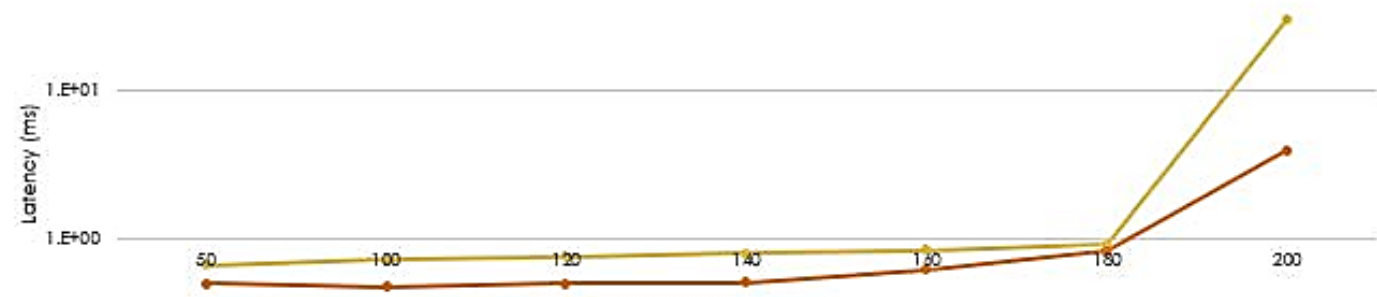

1 E-01 ONU load (MOps)

$\rightarrow$ RR-DSA $\rightarrow$ Opfimized $R R$

(a)

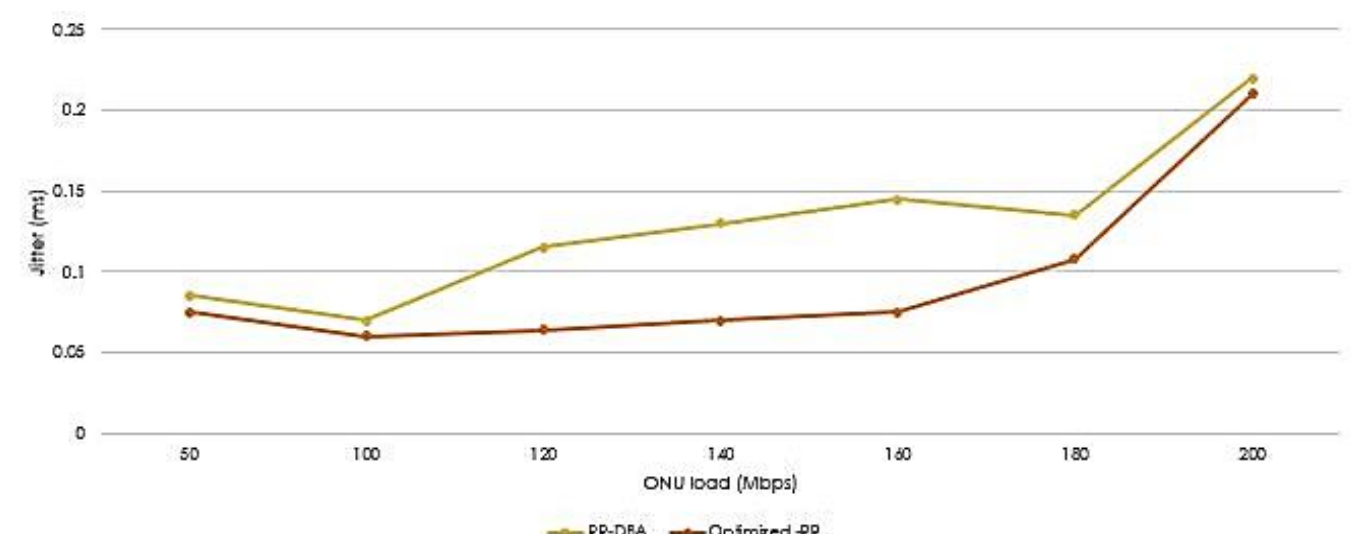

(b)

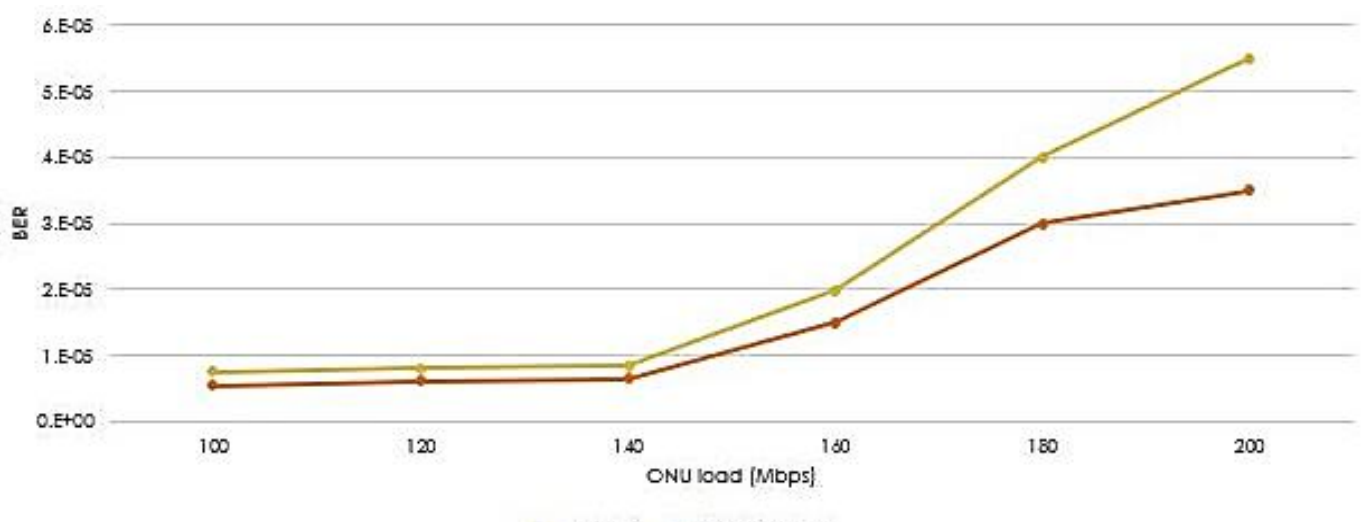

-RR-DBA -Opimized $R$ R

(c)

Figure 3. Performance comparison of original RR-DBA and the optimized-RR,

(a) Latency, (b) Jitter, (c) BER

\section{ACKNOWLEDGEMENTS}

This work was supported by the Pan African University Institute of Basic Science, Technology and Innovation PAUSTI, Kenya. 


\section{REFERENCES}

[1] I. A. Alimi, et al., "Toward an Efficient C-RAN Optical Fronthaul for the Future Networks: A Tutorial on Technologies, Requirements, Challenges, and Solutions," IEEE Communications Surveys and Tutorials, vol. 20, no. 1, pp. 708-769, 2018

[2] China Mobile, "C-RAN The Road Towards Green RAN," White Paper, China Mobile Research Institute, 2011.

[3] Radisys, "Evaluating Cloud RAN Implementation Scenarios," White Paper, 2014.

[4] H. L. Checko, et al., "Cloud Radio Access Network architecture. Towards 5G mobile networks Cloud," Technical University of Denmark, 2016.

[5] H. Lehrmann, et al., "Cloud RAN for Mobile Networks-a Technology Overview," IEEE Communications Surveys and Tutorials, vol. 17, no. 1, pp. 405-426, 2014.

[6] J. A. H. and D. L. L. G. O. Pérez, "Delay Analysis of Fronthaul Traffic in 5G Transport Networks," 2017 IEEE 17th International Conference of Ubiquitous Wireless Broadband, 2017, pp. 1-5.

[7] M. Waqar, et al., "A Performance Analysis of 5G Fronthaul Networks for Long-Distance Communications," 2019 Wireless Days, pp. 1-4, 2019.

[8] F. A. Khan, et al., "Performance Analysis of Cloud Radio Access Network," Norwegian University of Science and Technology, 2017.

[9] N. J. Gomes, et al., "Optical Fronthaul Options for Meeting 5G Requirements," 2018 20th International Conference of Transparent Optical Networks, 2018, pp. 1-4.

[10] NGMN Alliance, "Fronthaul Requirements for C-RAN," NGMN, 2015.

[11] J. Zhang, et al., "Performance Analysis of Optical Mobile Fronthaul for Cloud Radio Access Networks," Journal of Physics: Conference Series, vol. 910, 2017, pp. 1-6.

[12] G. O. Perez, et al., "Fronthaul network modeling and dimensioning meeting ultra-low latency requirements for 5G," Journal of Optical Communications and Networking, vol. 10, no. 6, pp. 573-581, 2018.

[13] A. Macho, et al., "Next-generation Optical Fronthaul Systems using Multicore Fiber Media," Journal of Lightwave Technology, vol. 34, no. 20, pp. 1-10, 2016.

[14] Fujitsu, "The Benefits of Cloud-RAN Architecture in Mobile Network Expansion," White Paper, 2014.

[15] N. P. Anthapadmanabhan, et al., "Mobile Fronthaul over Latency-Optimized Time Division Multiplexed Passive Optical Networks," 2015 IEEE International Conference on Communication Workshop (ICCW), London, 2015, pp. 62-67.

[16] H. Ou, et al., "Passive optical network range applicable to cost-effective mobile fronthaul," 2016 IEEE International Conference Communications (ICC 2016), 2016.

[17] Z. Tayq, et al., "Fronthaul Performance Demonstration in a WDM-PON-Based Convergent Network," EuCNC, pp. 1-5, 2016.

[18] Z. Ghebretensaé, et al., "Transmission Solutions and Architectures for Heterogeneous Networks Built as C-RANs," 2012 7th International ICST Conference on Communications and Networking in China, 2012, pp. 748-752.

[19] D. Nesset, "PON Roadmap," Journal of Optical Communications and Networking, vol. 9, no. 1, pp. 71-76, 2017.

[20] S. Kuwano, "Operator Perspective on Next-Generation Optical Access for Future Radio Access," 2014 IEEE International Conference on Communication Workshops, 2014, pp. 376-381.

[21] A. M. Mikaeil, et al., "Performance Evaluation of XG-PON Based Mobile Front-Haul Transport in Cloud-RAN Architecture," Journal of Optical Communications and Networking, vol. 9, no. 11, p. 984, 2017.

[22] S. Zhou, et al., "Low-Latency High-Efficiency Mobile Fronthaul With TDM-PON (Mobile-PON)," Journal of Optical Communications and Networking, vol. 10, no. 1, pp. 20-26, 2018.

[23] N. Shibata, et al., "Mobile front-haul employing Ethernet-based TDM - PON system for small cells," 2015 Optical Fiber Communications Conference and Exhibition (OFC), Los Angeles, 2015, pp. 7-9.

[24] N. Shibata, et al., "Performance Evaluation of Mobile Front-Haul Employing Ethernet-Based TDM-PON With IQ Data Compression [Invited]," IEEE/OSA Journal of Optical Communications and Networking, vol. 7, no. 11, pp. 3-9, 2015.

[25] A. Pizzinat, et al., "Things You Should Know About Fronthaul," Journal of Lightwave Technology, vol. 33, no. 5, pp. 1077-1083, 2015.

[26] T. Tashiro, et al., "A Novel DBA Scheme for TDM-PON based Mobile Fronthaul," OFC 2014, San Francisco, pp. 3-5, 2014.

[27] T. Horvath, et al., "Modified GIANT Dynamic Bandwidth Allocation Algorithm of NG-PON," Journal of Communications Software and Systems, vol. 13, no. 1, pp. 15-22, 2017.

[28] P. Sikora, et al., "Efficiency Tests of DBA Algorithms in XG-PON," Electronics, vol. 8, no. 7, p. 762, 2019.

[29] J. Prat, et al., "Sardana: an all-optical access-metro wdm/tdm-pon," 2014.

[30] L. Huawei Technologies Co., "XG(S)-PON (XG-PON and XGS-PON) Feature Guide," vol. 2208, no. 05, pp. 1-2, 2019. 


\section{BIOGRAPHIES OF AUTHORS}
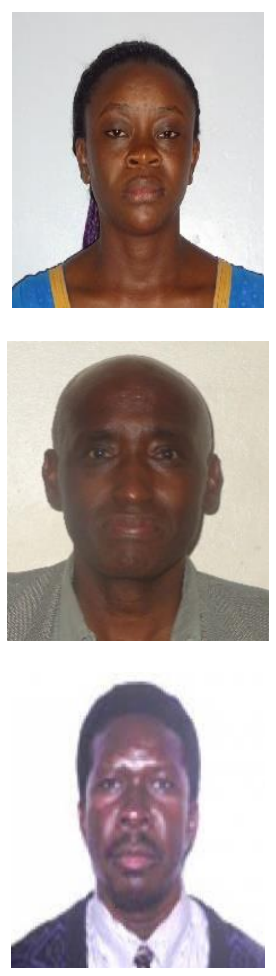

Ebude Carine Awasume is a holder of B. Eng and M. Eng in Telecommunications, Information and Communication Technology form the Faculty of Industrial Engineering, University of Douala, Cameroon. She is currently doing her PhD research in 5G C-RAN Fronthaul at the PAN African University, Nairobi. She is on Africa Union Scholarship. She can be reached by phone on +254743123216 and through E-mail ebude.carine@students.jkuat.ac.ke

Stephen Musyoski (PhD) B.Sc, M.Sc is an Associate Professor in the Department of Telecommunications and Information Engineering, Technical University of Kenya, Kenya.

V. K. Oduol (PhD) B. Eng. (Hons.), M. Eng., Ph.D. (McGill), MIEEE, MSPIE is a professor in the Department of Electrical \& Information Engineering, University of Nairobi, Nairobi, Kenya. 